\title{
An Operator Approach to the Al-Salam-Carlitz Polynomials
}

\author{
William Y. C. Chen ${ }^{1}$, Husam L. Saad ${ }^{2}$, and Lisa H. Sun ${ }^{3}$ \\ ${ }^{1,3}$ Center for Combinatorics, LPMC-TJKLC \\ Nankai University \\ Tianjin 300071, P.R. China \\ ${ }^{2}$ Department of Mathematics, College of Science, \\ Basrah University \\ Basrah, Iraq \\ ${ }^{1}$ chen@nankai.edu.cn, ${ }^{2}$ hus6274@hotmail.com, ${ }^{3}$ sunhui@nankai.edu.cn
}

\begin{abstract}
We present an operator approach to Rogers-type formulas and Mehler's formulas for the Al-Salam-Carlitz polynomials $U_{n}(x, y, a ; q)$. By using the $q$-exponential operator, we obtain a Rogers-type formula which leads to a linearization formula. With the aid of a bivariate augmentation operator, we get a simple derivation of Mehler's formula due to by Al-Salam and Carlitz, which requires a terminating condition on a ${ }_{3} \phi_{2}$ series. By means of the Cauchy companion augmentation operator, we obtain Mehler's formula in a similar form, but it does not need the terminating condition. We also give several identities on the generating functions for products of the Al-Salam-Carlitz polynomials which are extensions of formulas for Rogers-Szegö polynomials.
\end{abstract}

Keywords: Al-Salam-Carlitz polynomial, the $q$-exponential operator, the homogeneous $q$ shift operator, the Cauchy companion operator, the Rogers-type formula, Mehler's formula

AMS Classification: 33D45, 05A30

\section{Introduction}

The Al-Salam-Carlitz polynomials are $q$-orthogonal polynomials which arise in many applications such as the $q$-harmonic oscillator, theta functions, quantum groups and coding theory; see for example $[4,5,15,16]$. This paper presents an operator approach to the Rogers-type formulas and Mehler's formulas for the Al-Salam-Carlitz polynomials. These polynomials are a generalization of the classical Rogers-Szegö polynomials which have been extensively studied, see for example $[6-8,10,13]$. There are two classical formulas concerning the RogersSzegö polynomials, namely, Mehler's formula and the Rogers formula, in connection with the Poisson kernel formula and the linearization formula.

It is natural to study the Rogers-type formulas and Mehler's formulas beyond the RogersSzegö polynomials. In fact, Mehler's formula for the Al-Salam-Carlitz polynomials has been 
derived by Al-Salam and Carlitz [2], which requires a terminating condition on a ${ }_{3} \phi_{2}$ series as mentioned by Askey and Suslov [4]. Using our operator approach, we deduce a new formula in a similar form, but it does not involve the terminating condition. We also derive some Rogers-type formulas, one of which leads to a linearization formula. In addition, we obtain several identities on the generating functions of products of the Al-Salam-Carlitz polynomials as extensions of the formulas for the Rogers-Szegö polynomials.

We adopt the common notation on $q$-series in Gasper and Rahman [15]. The set of integers is denoted by $\mathbb{Z}$. Throughout this paper, $q$ is a fixed nonzero complex number with $|q|<1$. The $q$-shifted factorial is defined for any complex parameter $a$ by

$$
(a ; q)_{\infty}=\prod_{k=0}^{\infty}\left(1-a q^{k}\right) \quad \text { and } \quad(a ; q)_{n}=\frac{(a ; q)_{\infty}}{\left(a q^{n} ; q\right)_{\infty}}, \quad n \in \mathbb{Z} .
$$

We shall use the compact notation

$$
\left(a_{1}, a_{2}, \ldots, a_{m} ; q\right)_{n}=\left(a_{1} ; q\right)_{n}\left(a_{2} ; q\right)_{n} \cdots\left(a_{m} ; q\right)_{n}, \quad \text { for } n \in \mathbb{Z} \text { or } n=\infty .
$$

The $q$-binomial coefficient is given by

$$
\left[\begin{array}{c}
n \\
k
\end{array}\right]=\frac{(q ; q)_{n}}{(q ; q)_{n-k}(q ; q)_{k}} .
$$

The basic hypergeometric series ${ }_{r} \phi_{s}$ are defined as follows,

$$
{ }_{r} \phi_{s}\left[\begin{array}{c}
a_{1}, a_{2}, \ldots, a_{r} \\
b_{1}, b_{2}, \ldots, b_{s}
\end{array} ; q, x\right]=\sum_{n=0}^{\infty} \frac{\left(a_{1}, a_{2}, \ldots, a_{r} ; q\right)_{n}}{\left(q, b_{1}, b_{2}, \ldots, b_{s} ; q\right)_{n}}\left[(-1)^{n} q^{\left(\begin{array}{c}
n \\
2
\end{array}\right)}\right]^{1+s-r} x^{n} .
$$

This paper is primarily concerned with the Al-Salam-Carlitz polynomials $U_{n}(x, y, a ; q)$ which can be defined in terms of a ${ }_{2} \phi_{1}$ series

$$
U_{n}(x, y, a ; q)=(-a)^{n} q^{\left(\begin{array}{c}
n \\
2
\end{array}\right)}{ }_{2} \phi_{1}\left(\begin{array}{c}
q^{-n}, y / x \\
0
\end{array} ; q, \frac{q x}{a}\right) .
$$

The following generating function for the the Al-Salam-Carlitz polynomials has been given by Al-Salam and Carlitz [2],

$$
\sum_{n=0}^{\infty} U_{n}(x, y, a ; q) \frac{t^{n}}{(q ; q)_{n}}=\frac{(a t, y t ; q)_{\infty}}{(x t ; q)_{\infty}}
$$

where $|x t|<1$. Since that the right-hand side of (1.3) is symmetric in $a$ and $y$, the polynomials $U_{n}(x, y, a ; q)$ are symmetric in $a$ and $y$, that is,

$$
U_{n}(x, y, a ; q)=U_{n}(x, a, y ; q) .
$$

This symmetry property will be used later.

In terms of of the Cauchy polynomials

$$
P_{n}(x, y)=(x-y)(x-q y) \cdots\left(x-q^{n-1} y\right)
$$


with the generating function

$$
\sum_{n=0}^{\infty} P_{n}(x, y) \frac{t^{n}}{(q ; q)_{n}}=\frac{(y t ; q)_{\infty}}{(x t ; q)_{\infty}}, \quad|x t|<1,
$$

the Al-Salam-Carlitz polynomials can be expressed as

$$
U_{n}(x, y, a ; q)=\sum_{k=0}^{n}\left[\begin{array}{l}
n \\
k
\end{array}\right](-1)^{k} q^{\left(\begin{array}{c}
k \\
2
\end{array}\right) a^{k} P_{n-k}(x, y) .}
$$

The above definition is essentially the same as the original definition of the Al-Salam-Carlitz polynomials $u_{n}^{(a)}(x ; q)$,

$$
u_{n}^{(a)}(x ; q)=(-a)^{n} q^{\left(\begin{array}{c}
n \\
2
\end{array}\right)}{ }_{2} \phi_{1}\left(\begin{array}{c}
q^{-n}, x^{-1} \\
0
\end{array} ; q, \frac{q x}{a}\right) .
$$

Clearly, we have the following relation

$$
U_{n}(x, y, a ; q)=y^{n} u_{n}^{(a / y)}(x / y ; q) .
$$

The Al-Salam-Carlitz polynomials are related to several $q$-orthogonal polynomials, such as the $q$-Bessel polynomials $B_{n}(x, b ; q)$ due to Abdi [1], and the Stieltjes-Wigert polynomials $S_{n}(x ; q)[17$, p. 116]. In particular, the Al-Salam-Carlitz polynomials are connected to the bivariate Rogers-Szegö polynomials [11]

$$
h_{n}(x, y \mid q)=\sum_{k=0}^{n}\left[\begin{array}{l}
n \\
k
\end{array}\right] P_{k}(x, y),
$$

which have the generating function

$$
\sum_{n=0}^{\infty} h_{n}(x, y \mid q) \frac{t^{n}}{(q ; q)_{n}}=\frac{(y t ; q)_{\infty}}{(t, x t ; q)_{\infty}}, \quad|t|<1,|x t|<1 .
$$

On the other hand, although the Al-Salam-Carlitz polynomials can be expressed in terms of the bivariate Rogers-Szegö polynomials

$$
U_{n}(x, y, a ; q)=(-1)^{n} q^{\left(\begin{array}{c}
n \\
2
\end{array}\right)} a^{n} h_{n}\left(\frac{y}{a}, \frac{x}{a} \mid q^{-1}\right),
$$

as noted by Carlitz [8], it is often happens that an infinite $q$-series identity no longer holds when $q$ is replaced by $q^{-1}$. In fact, it turns out to be the case for the Rogers formula and Mehler's formula for the polynomials $h_{n}(x, y \mid q)$. This suggests that there is a need for a direct approach to deal with the Al-Salam-Carlitz polynomials, and it is our hope to serve this purpose.

This paper is organized as follows. In Section 2, we give an overview of the $q$-exponential operator $T\left(b D_{q}\right)$, and derive a Rogers-type formula for $U_{n}(x, y, a ; q)$ which leads to a linearization formula. In Section 3, we construct a homogeneous $q$-shift operator $\mathbb{F}\left(a D_{x y}\right)$ and apply it to derive Mehler's formula under the terminating condition. In Section 4, we make use of the Cauchy companion operator to obtain two Rogers-type formulas and Mehler's formula without the terminating condition. In the last section, we provide four generating function identities for products of $U_{n}(x, y, a ; q)$. 


\section{A Rogers-Type Formula}

In this section, we give a Rogers-type formula for the Al-Salam-Carlitz polynomials $U_{n}(x, y, a ; q)$ by using the $q$-exponential operator $T\left(b D_{q}\right)$. As a consequence, we obtain a linearization formula for $U_{n}(x, y, a ; q)$.

The $q$-differential operator, or the $q$-derivative, acting on the variable $a$, is defined by

$$
D_{q}\{f(a)\}=\frac{f(a)-f(a q)}{a} .
$$

The $q$-shift operator, denoted by $\eta$, is defined by

$$
\eta\{f(a)\}=f(a q) \text { and } \eta^{-1}\{f(a)\}=f\left(a q^{-1}\right),
$$

see, for example, $[3,18]$. The operator $\theta$ is defined by

$$
\theta=\eta^{-1} D_{q}
$$

see Roman [18]. Recall the $q$-Leibniz rule for $D_{q}$, see [18],

$$
D_{q}^{n}\{f(a) g(a)\}=\sum_{k=0}^{n}\left[\begin{array}{l}
n \\
k
\end{array}\right] q^{k(k-n)} D_{q}^{k}\{f(a)\} D_{q}^{n-k}\left\{g\left(q^{k} a\right)\right\} .
$$

By convention, $D_{q}^{0}$ is understood as the identity, that is, $D_{q}^{0}\{f(a)\}=f(a)$. Chen and Liu [12] introduced the following two $q$-exponential operators

$$
T\left(b D_{q}\right)=\sum_{n=0}^{\infty} \frac{\left(b D_{q}\right)^{n}}{(q ; q)_{n}} \quad \text { and } \quad E(b \theta)=\sum_{n=0}^{\infty} \frac{q^{\left(\begin{array}{c}
n \\
2
\end{array}\right)(b \theta)^{n}}}{(q ; q)_{n}}
$$

for proving basic hypergeometric identities from their special cases. This method is called parameter augmentation. The following lemma for the $q$-exponential operator $T\left(b D_{q}\right)$ is easy to verify.

Lemma 2.1. We have

$$
T\left(b D_{q}\right)\left\{a^{n}\right\}=\sum_{k=0}^{n}\left[\begin{array}{l}
n \\
k
\end{array}\right] b^{k} a^{n-k}
$$

From the $q$-Leibniz rule for $D_{q}$, Zhang and Wang [19] obtain the following identity.

Lemma 2.2. Let $n$ be a nonnegative integer. Then

$$
D_{q}^{n}\left\{\frac{(a t ; q)_{\infty}}{(a v ; q)_{\infty}}\right\}=v^{n}(t / v ; q)_{n} \frac{\left(a t q^{n} ; q\right)_{\infty}}{(a v ; q)_{\infty}} .
$$

Based on the above relation, we obtain the following formula.

Lemma 2.3. We have

$$
T\left(b D_{q}\right)\left\{\frac{(a s, a t ; q)_{\infty}}{(a v ; q)_{\infty}}\right\}=\frac{(a s, a t ; q)_{\infty}}{(a v ; q)_{\infty}} \sum_{k=0}^{\infty} \frac{(-1)^{k} q^{\left(\begin{array}{c}
k \\
2
\end{array}\right)(a v ; q)_{k}(b s)^{k}}}{(q ; q)_{k}(a s, a t ; q)_{k}}{ }_{2} \phi_{1}\left(\begin{array}{c}
t / v, 0 \\
a t q^{k}
\end{array} ; q, b v\right) .
$$


Proof. By the definition of $T\left(b D_{q}\right)$ and the $q$-Leibniz rule for $D_{q}$, we have

$$
\begin{aligned}
T\left(b D_{q}\right) & \left\{\frac{(a s, a t ; q)_{\infty}}{(a v ; q)_{\infty}}\right\}=\sum_{n=0}^{\infty} \frac{b^{n}}{(q ; q)_{n}} D_{q}^{n}\left\{\frac{(a s, a t ; q)_{\infty}}{(a v ; q)_{\infty}}\right\} \\
& =\sum_{n=0}^{\infty} \frac{b^{n}}{(q ; q)_{n}} \sum_{k=0}^{n}\left[\begin{array}{l}
n \\
k
\end{array}\right] q^{k(k-n)} D_{q}^{k}\left\{(a s ; q)_{\infty}\right\} D_{q}^{n-k}\left\{\frac{\left(a t q^{k} ; q\right)_{\infty}}{\left(a v q^{k} ; q\right)_{\infty}}\right\} \\
& =\sum_{n=0}^{\infty} \frac{b^{n}}{(q ; q)_{n}} \sum_{k=0}^{n}\left[\begin{array}{l}
n \\
k
\end{array}\right] q^{k(k-n)}(-1)^{k} q^{\left(\begin{array}{c}
k \\
2
\end{array}\right) s^{k}\left(a s q^{k} ; q\right)_{\infty}\left(v q^{k}\right)^{n-k}(t / v ; q)_{n-k}} \frac{\left(a t q^{n} ; q\right)_{\infty}}{\left(a v q^{k} ; q\right)_{\infty}} \\
& =\frac{(a s, a t ; q)_{\infty}}{(a v ; q)_{\infty}} \sum_{n=0}^{\infty} \frac{b^{n}}{(q ; q)_{n}} \sum_{k=0}^{n}\left[\begin{array}{l}
n \\
k
\end{array}\right](-1)^{k} q^{\left(\begin{array}{c}
k \\
2
\end{array}\right)} s^{k} \frac{(a v ; q)_{k}(t / v ; q)_{n-k} v^{n-k}}{(a s ; q)_{k}(a t ; q)_{n}} \\
= & \frac{(a s, a t ; q)_{\infty}}{(a v ; q)_{\infty}} \sum_{k=0}^{\infty} \frac{(-1)^{k} q^{\left(\begin{array}{c}
k \\
2
\end{array}\right)(a v ; q)_{k}(b s)^{k}}}{(q, a s, a t ; q)_{k}} \sum_{n=0}^{\infty} \frac{(t / v ; q)_{n}(b v)^{n}}{(q ; q)_{n}\left(a t q^{k} ; q\right)_{n}} \\
= & \frac{(a s, a t ; q)_{\infty}}{(a v ; q)_{\infty}} \sum_{k=0}^{\infty} \frac{(-1)^{k} q^{\left(\begin{array}{c}
k \\
2
\end{array}\right)(a v ; q)_{k}(b s)^{k}}}{(q ; q)_{k}(a s, a t ; q)_{k}}{ }_{2} \phi_{1}\left(\begin{array}{c}
t / v, 0 \\
\left.a t q^{k} ; q, b v\right)
\end{array}\right.
\end{aligned}
$$

This completes the proof.

Now we are ready to present a Rogers-type formula for the polynomials $U_{n}(x, y, a ; q)$.

Theorem 2.4. We have

$$
\begin{aligned}
& \sum_{n=0}^{\infty} \sum_{m=0}^{\infty} U_{n+m}(x, y, a ; q) \frac{t^{n}}{(q ; q)_{n}} \frac{s^{m}}{(q ; q)_{m}} \\
& \quad=\frac{(a s, y s ; q)_{\infty}}{(x s ; q)_{\infty}} \sum_{k=0}^{\infty} \frac{(-1)^{k} q^{\left(\begin{array}{c}
k \\
2
\end{array}\right)(x s ; q)_{k}(a t)^{k}}}{(q ; q)_{k}(a s, y s ; q)_{k}}{ }_{2} \phi_{1}\left(\begin{array}{c}
y / x, 0 \\
y s q^{k}
\end{array} ; q, x t\right)
\end{aligned}
$$

provided that $|x s|<1$.

Proof. Setting $m \rightarrow m-n$, exchanging the order of the sum on the left hand side of (2.4), and applying the operator identity (2.2), we obtain

$$
\begin{aligned}
\sum_{n=0}^{\infty} & \sum_{m=0}^{\infty} U_{n+m}(x, y, a ; q) \frac{t^{n}}{(q ; q)_{n}} \frac{s^{m}}{(q ; q)_{m}} \\
& =\sum_{n=0}^{\infty} \sum_{m=n}^{\infty} U_{m}(x, y, a ; q) \frac{t^{n}}{(q ; q)_{n}} \frac{s^{m-n}}{(q ; q)_{m-n}}=\sum_{m=0}^{\infty} \frac{U_{m}(x, y, a ; q)}{(q ; q)_{m}} \sum_{n=0}^{m}\left[\begin{array}{c}
m \\
n
\end{array}\right] t^{n} s^{m-n} \\
& =\sum_{m=0}^{\infty} \frac{U_{m}(x, y, a ; q)}{(q ; q)_{m}} T\left(t D_{q}\right)\left\{s^{m}\right\}=T\left(t D_{q}\right)\left\{\sum_{m=0}^{\infty} U_{m}(x, y, a ; q) \frac{s^{m}}{(q ; q)_{m}}\right\} \quad(|x s|<1) \\
& =T\left(t D_{q}\right)\left\{\frac{(a s, y s ; q)_{\infty}}{(x s ; q)_{\infty}}\right\},
\end{aligned}
$$

where $D_{q}$ acts on the parameter $s$. Applying Lemma 2.3, we complete the proof. 
From the above Rogers-type formula, we obtain the following linearization formula for $U_{n}(x, y, a ; q)$.

Theorem 2.5. For $n, m \geq 0$, we have

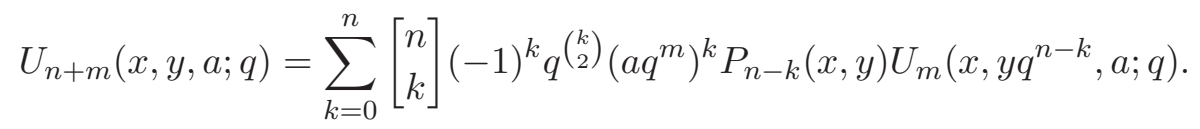

Proof. Rewrite the Rogers-type formula (2.4) as follows

$$
\begin{aligned}
\sum_{n=0}^{\infty} & \sum_{m=0}^{\infty} U_{n+m}(x, y, a ; q) \frac{t^{n}}{(q ; q)_{n}} \frac{s^{m}}{(q ; q)_{m}} \\
& =\sum_{k=0}^{\infty} \frac{(-1)^{k} q^{\left(\begin{array}{c}
k \\
2
\end{array}\right)(a t)^{k}}}{(q ; q)_{k}} \sum_{n=0}^{\infty} \frac{(y / x ; q)_{n}}{(q ; q)_{n}}(x t)^{n} \frac{\left(a s q^{k}, y s q^{n+k} ; q\right)_{\infty}}{\left(x s q^{k} ; q\right)_{\infty}} \\
& =\sum_{k=0}^{\infty} \frac{(-1)^{k} q^{\left(\begin{array}{c}
k \\
2
\end{array}\right)(a t)^{k}}}{(q ; q)_{k}} \sum_{n=0}^{\infty} \frac{(y / x ; q)_{n}}{(q ; q)_{n}}(x t)^{n} \sum_{l=0}^{\infty} U_{l}\left(x, y q^{n}, a ; q\right) \frac{\left(s q^{k}\right)^{l}}{(q ; q)_{l}}
\end{aligned}
$$

Equating the coefficients of $t^{n} s^{m}$ in the above equation, the desired identity follows.

\section{Mehler's Formula}

In this section, we aim to introduce the homogeneous $q$-shift operator which can be used to give a simple derivation of Mehler's formula for $U_{n}(x, y, a ; q)$ due to Al-Salam and Carlitz.

Recall that the homogeneous $q$-difference operator $D_{x y}$ introduced by Chen, Fu and Zhang $[11]$ is given by

$$
D_{x y}\{f(x, y)\}=\frac{f\left(x, q^{-1} y\right)-f(q x, y)}{x-q^{-1} y} .
$$

Based on this operator $D_{x y}$, we construct the following homogeneous $q$-shift operator

$$
\mathbb{F}\left(a D_{x y}\right)=\sum_{n=0}^{\infty} \frac{(-1)^{n} q^{\left(\begin{array}{c}
n \\
2
\end{array}\right)\left(a D_{x y}\right)^{n}}}{(q ; q)_{n}} .
$$

The $q$-difference operator $D_{x y}$ has the following basic properties.

Proposition 3.1. We have

$$
\begin{aligned}
D_{x y}^{k}\left\{P_{n}(x, y)\right\} & =\frac{(q ; q)_{n}}{(q ; q)_{n-k}} P_{n-k}(x, y), \\
D_{x y}^{k}\left\{\frac{(y t ; q)_{\infty}}{(x t ; q)_{\infty}}\right\} & =t^{k} \frac{(y t ; q)_{\infty}}{(x t ; q)_{\infty}} .
\end{aligned}
$$

Invoking (3.3), the Al-Salam-Carlitz polynomials $U_{n}(x, y, a ; q)$ can be expressed in terms of the homogeneous $q$-shift operator $\mathbb{F}\left(a D_{x y}\right)$. 
Theorem 3.2. We have

$$
U_{n}(x, y, a ; q)=\mathbb{F}\left(a D_{x y}\right)\left\{P_{n}(x, y)\right\}
$$

By using (3.4), it is easy to derive the following relation.

Proposition 3.3. We have

$$
\mathbb{F}\left(a D_{x y}\right)\left\{\frac{(y t ; q)_{\infty}}{(x t ; q)_{\infty}}\right\}=\frac{(a t, y t ; q)_{\infty}}{(x t ; q)_{\infty}} .
$$

Combining (3.5) and (3.6), the generating function of $U_{n}(x, y, a ; q)$ can be derived as follows,

$$
\begin{aligned}
\sum_{n=0}^{\infty} U_{n}(x, y, a ; q) \frac{t^{n}}{(q ; q)_{n}} & =\mathbb{F}\left(a D_{x y}\right)\left\{\sum_{n=0}^{\infty} P_{n}(x, y) \frac{t^{n}}{(q ; q)_{n}}\right\} \quad(|x t|<1) \\
& =\mathbb{F}\left(a D_{x y}\right)\left\{\frac{(y t ; q)_{\infty}}{(x t ; q)_{\infty}}\right\}=\frac{(a t, y t ; q)_{\infty}}{(x t ; q)_{\infty}}
\end{aligned}
$$

The following identity will be used to derive Mehler's formula.

Theorem 3.4. Assume $|x s|<1$. We have

$$
\mathbb{F}\left(a D_{x y}\right)\left\{\frac{P_{n}(x, y)(y s ; q)_{\infty}}{(y s ; q)_{n}(x s ; q)_{\infty}}\right\}=\frac{\left(y s q^{n}, a s ; q\right)_{\infty}}{(x s ; q)_{\infty}} \sum_{k=0}^{n}\left[\begin{array}{l}
n \\
k
\end{array}\right](-1)^{k} q^{\left(\begin{array}{c}
k \\
2
\end{array}\right)} \frac{(x s ; q)_{k}(y / x ; q)_{n-k}}{(a s ; q)_{k}} x^{n-k} a^{k}
$$

Proof. Applying (3.5), the left hand side of the Rogers-type formula (2.4) equals

$$
\begin{aligned}
\mathbb{F}\left(a D_{x y}\right)\left\{\sum_{n=0}^{\infty} \sum_{m=0}^{\infty} P_{n+m}(x, y) \frac{t^{n}}{(q ; q)_{n}} \frac{s^{m}}{(q ; q)_{m}}\right\} \\
=\mathbb{F}\left(a D_{x y}\right)\left\{\sum_{n=0}^{\infty} P_{n}(x, y) \frac{t^{n}}{(q ; q)_{n}} \sum_{m=0}^{\infty} P_{m}\left(x, q^{n} y\right) \frac{s^{m}}{(q ; q)_{m}}\right\} \quad(|x s|<1) \\
=\mathbb{F}\left(a D_{x y}\right)\left\{\sum_{n=0}^{\infty} P_{n}(x, y) \frac{t^{n}}{(q ; q)_{n}} \frac{\left(y q^{n} s ; q\right)_{\infty}}{(x s ; q)_{\infty}}\right\} \\
=\sum_{n=0}^{\infty} \mathbb{F}\left(a D_{x y}\right)\left\{\frac{P_{n}(x, y)(y s ; q)_{\infty}}{(y s ; q)_{n}(x s ; q)_{\infty}}\right\} \frac{t^{n}}{(q ; q)_{n}} .
\end{aligned}
$$

On the other hand, the right hand side of (2.4) can be restated as

$$
\frac{(a s, y s ; q)_{\infty}}{(x s ; q)_{\infty}} \sum_{k=0}^{\infty} \frac{(-1)^{k} q^{\left(\begin{array}{c}
k \\
2
\end{array}\right)}(x s ; q)_{k}(a t)^{k}}{(q ; q)_{k}(a s, y s ; q)_{k}} \sum_{l=0}^{\infty} \frac{(y / x ; q)_{l}}{\left(q, y s q^{k} ; q\right)_{l}}(x t)^{l} .
$$

Equating the coefficients of $t^{n}$ in (3.8) and (3.9), we complete the proof. 
Applying the above operator identity, we obtain Mehler's formula involving a terminating ${ }_{3} \phi_{2}$ series.

Theorem 3.5. We have

$$
\begin{aligned}
& \sum_{n=0}^{\infty}(-1)^{n} q^{-\left(\begin{array}{c}
n \\
2
\end{array}\right)} U_{n}(x, y, a ; q) U_{n}(u, v, b ; q) \frac{t^{n}}{(q ; q)_{n}} \\
& \quad=\frac{(a b t, y b t, a v t ; q)_{\infty}}{(x b t, a u t ; q)_{\infty}}{ }_{3} \phi_{2}\left(\begin{array}{c}
y / x, v / u, q / a b t \\
q / x b t, q / a u t
\end{array} ; q, q\right)
\end{aligned}
$$

where $y / x=q^{-r}$ or $v / u=q^{-r}$ for a nonnegative integer $r$, and $\max \left\{\left|x b t q^{-r}\right|,\left|a u t q^{-r}\right|\right\}<1$.

Proof. Using (3.5), we find

$$
\begin{aligned}
& \sum_{n=0}^{\infty}(-1)^{n} q^{-\left(\begin{array}{c}
n \\
2
\end{array}\right)} U_{n}(x, y, a ; q) U_{n}(u, v, b ; q) \frac{t^{n}}{(q ; q)_{n}} \\
& =\mathbb{F}\left(a D_{x y}\right)\left\{\sum_{n=0}^{\infty}(-1)^{n} q^{-\left(\begin{array}{c}
n \\
2
\end{array}\right)} P_{n}(x, y) U_{n}(u, v, b ; q) \frac{t^{n}}{(q ; q)_{n}}\right\} \\
& =\mathbb{F}\left(a D_{x y}\right)\left\{\sum_{n=0}^{\infty}(-1)^{n} q^{-\left(\begin{array}{c}
n \\
2
\end{array}\right)} P_{n}(x, y)\left(\sum_{k=0}^{n}\left[\begin{array}{l}
n \\
k
\end{array}\right](-1)^{k} q^{\left.\left(\begin{array}{c}
k \\
2
\end{array}\right) b^{k} P_{n-k}(u, v)\right)} \frac{t^{n}}{(q ; q)_{n}}\right\}\right. \\
& =\mathbb{F}\left(a D_{x y}\right)\left\{\sum_{n=0}^{\infty} \frac{(-1)^{n} q^{-\left(\begin{array}{c}
n \\
2
\end{array}\right)} P_{n}(u, v) P_{n}(x, y) t^{n}}{(q ; q)_{n}} \sum_{k=0}^{\infty} P_{k}\left(x, q^{n} y\right) \frac{\left(b t q^{-n}\right)^{k}}{(q ; q)_{k}}\right\} \text {. }
\end{aligned}
$$

The terminating condition $v / u=q^{-r}$ or $y / x=q^{-r}$ implies that the first sum in (3.11) is finite. Utilizing (1.5), we see that (3.11) equals

$$
\sum_{n=0}^{\infty} \frac{(-1)^{n} q^{-\left(\begin{array}{c}
n \\
2
\end{array}\right)} P_{n}(u, v) t^{n}}{(q ; q)_{n}} \mathbb{F}\left(a D_{x y}\right)\left\{\frac{P_{n}(x, y)}{\left(y b t q^{-n} ; q\right)_{n}} \frac{\left(y b t q^{-n} ; q\right)_{\infty}}{\left(x b t q^{-n} ; q\right)_{\infty}}\right\} .
$$

Applying (3.7) with $s \rightarrow b t q^{-n}$, the above sum equals

$$
\begin{aligned}
& \sum_{n=0}^{\infty} \frac{(-1)^{n} q^{-\left(\begin{array}{l}
n \\
2
\end{array}\right)} P_{n}(u, v) t^{n}}{(q ; q)_{n}} \frac{\left(y b t, a b t q^{-n} ; q\right)_{\infty}}{\left(x b t q^{-n} ; q\right)_{\infty}} \sum_{k=0}^{n}\left[\begin{array}{l}
n \\
k
\end{array}\right](-1)^{k} q^{\left(\begin{array}{c}
k \\
2
\end{array}\right)} \frac{\left(x b t q^{-n} ; q\right)_{k}(y / x ; q)_{n-k}}{\left(a b t q^{-n} ; q\right)_{k}} x^{n-k} a^{k}
\end{aligned}
$$

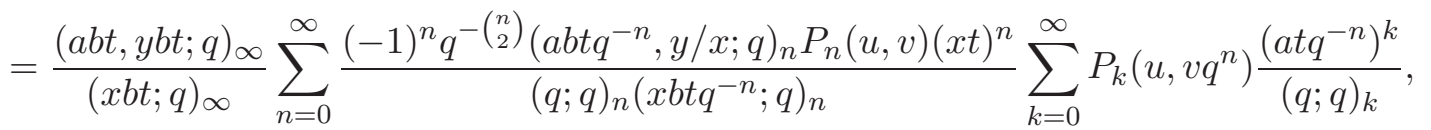

Under the terminating condition, the above sum further simplifies to the right hand side of (3.10). This completes the proof.

We remark that the second sums in (3.11) and (3.12) do not converge when $n$ tends to infinity. To avoid this problem, we may restrict our attention to the case that $v / u=q^{-r}$ or $y / x=q^{-r}$, where $r$ is a nonnegative integer, as noticed by Askey and Suslov [4] and Fang [14]. 
Owing to the symmetry property and the relation (1.8), Mehler's formula for the AlSalam-Carlitz polynomials $u_{n}^{(a)}(x ; q)$ given by Al-Salam and Carlitz [2] can be recovered from the above theorem by setting $y / a \rightarrow a, x / a \rightarrow x, v / b \rightarrow b, u / b \rightarrow y,-a b t \rightarrow t$.

Corollary 3.5.1 (Mehler's formula for $\left.u_{n}^{(a)}(x \mid q)\right)$.

$$
\sum_{n=0}^{\infty} q^{-\left(\begin{array}{c}
n \\
2
\end{array}\right)} u_{n}^{(a)}(x ; q) u_{n}^{(b)}(y ; q) \frac{t^{n}}{(q ; q)_{n}}=\frac{(-t,-a t,-b t ; q)_{\infty}}{(-x t,-y t ; q)_{\infty}}{ }_{3} \phi_{2}\left(\begin{array}{c}
a / x, b / y,-q / t \\
-q / x t,-q / y t
\end{array} ; q, q\right)
$$

where $a / x=q^{-r}$ or $b / y=q^{-r}$ for a nonnegative integer $r$, and $\max \left\{\left|x t q^{-r}\right|,\left|y t q^{-r}\right|\right\}<1$.

\section{The Cauchy Companion Operator}

In this section, we apply the Cauchy companion operator defined by Chen [9] to derive the Rogers-type formulas and Mehler's formula without the terminating condition. Recall that the Cauchy augmentation operator is defined by Chen and $\mathrm{Gu}[10]$,

$$
T\left(a, b ; D_{q}\right)=\sum_{n=0}^{\infty} \frac{(a ; q)_{n}}{(q ; q)_{n}}\left(b D_{q}\right)^{n}
$$

Moreover, Chen [9] introduced the Cauchy companion operator

$$
E(a, b ; \theta)=\sum_{n=0}^{\infty} \frac{(a ; q)_{n}(-b \theta)^{n}}{(q ; q)_{n}}
$$

As observed by Chen [9], when one applies $E(a, b ; \theta)$ to the product $(c s, c t ; q)_{\infty} /(c v ; q)_{\infty}$, one does not get a valid identity by directly using $q$-Leibniz rule because of the convergence consideration. Instead, we may use the following expansion for $D_{q}^{n}$

$$
D_{q}^{n}\{f(c)\}=c^{-n} q^{-\left(\begin{array}{c}
n \\
2
\end{array}\right)} \sum_{k=0}^{n}(-1)^{k}\left[\begin{array}{l}
n \\
k
\end{array}\right] q^{\left(\begin{array}{c}
n-k \\
2
\end{array}\right)} f\left(c q^{k}\right) .
$$

In this way, we can deduce an alternative expansion for $E(a, b ; \theta)$ which is convergent [9]

$$
E(a, b ; \theta)\{f(c)\}=\frac{(a b q / c ; q)_{\infty}}{(b q / c ; q)_{\infty}} \sum_{k=0}^{\infty} \frac{(a ; q)_{k} f\left(c q^{-k}\right) q^{\left(\begin{array}{c}
k \\
2
\end{array}\right)}}{(q, a b q / c ; q)_{k}}\left(-\frac{b q}{c}\right)^{k}
$$

where $|b q / c|<1$. Furthermore, we will be able to derive the Rogers-type formulas and a Mehler's formula without the terminating condition based on the following operator identities established by Chen [9].

Proposition 4.1. Assume that the operator $E(a, b ; \theta)$ acts on the parameter $c$, then

$$
E(a, b ; \theta)\left\{c^{n}\right\}=\sum_{k=0}^{n}\left[\begin{array}{l}
n \\
k
\end{array}\right](a ; q)_{k}(-b q)^{k} c^{n-k} q^{\left(\begin{array}{c}
k \\
2
\end{array}\right)} q^{-n k}, \quad(n \geq 0),
$$




$$
\begin{aligned}
& E(a, b ; \theta)\left\{\frac{(c t ; q)_{\infty}}{(c v ; q)_{\infty}}\right\}=\frac{(c t ; q)_{\infty}}{(c v ; q)_{\infty}}{ }_{2} \phi_{1}\left(\begin{array}{c}
a, t / v \\
q / c v
\end{array} ; q, \frac{b q}{c}\right), \quad(|b q / c|<1) \\
& E(a, b ; \theta)\left\{\frac{(c s, c t ; q)_{\infty}}{(c v ; q)_{\infty}}\right\}=\frac{(a b q / c, c s, c t ; q)_{\infty}}{(b q / c, c v ; q)_{\infty}} \phi_{2}\left(\begin{array}{c}
a, q / c s, q / c t \\
a b q / c, q / c v
\end{array} ; q, \frac{b s t}{v}\right) \\
& \quad(\max \{|b q / c|,|b s t / v|\}<1)
\end{aligned}
$$

In the light of the property (4.3), we obtain the following operator representation of $U_{n}(x, y, a ; q)$.

Theorem 4.2. Assume that the operator $E(y / x, x ; \theta)$ acts on the parameter a, then

$$
E(y / x, x ; \theta)\left\{(-1)^{n} q^{\left.\left(\begin{array}{c}
n \\
2
\end{array}\right) a^{n}\right\}=U_{n}(x, y, a ; q) .}\right.
$$

The above operator identity leads to another Rogers-type formula for the Al-Salam-Carlitz polynomials.

Theorem 4.3. We have

$$
\sum_{n=0}^{\infty} \sum_{m=0}^{\infty}(-1)^{n} q^{-\left(\begin{array}{c}
n \\
2
\end{array}\right)-n m} U_{n+m}(x, y, a ; q) \frac{t^{n}}{(q ; q)_{n}} \frac{s^{m}}{(q ; q)_{m}}=\frac{(a s ; q)_{\infty}}{(a t ; q)_{\infty}}{ }_{2} \phi_{1}\left(\begin{array}{c}
y / x, s / t \\
q / a t
\end{array} ; q, \frac{x q}{a}\right)
$$

where $\max \{|a t|,|x q / a|\}<1$.

Proof. By (4.6), the left hand side of (4.7) can be written as

$$
\begin{aligned}
& \sum_{n=0}^{\infty} \sum_{m=0}^{\infty}(-1)^{n} q^{-\left(\begin{array}{c}
n \\
2
\end{array}\right)-n m} U_{n+m}(x, y, a ; q) \frac{t^{n}}{(q ; q)_{n}} \frac{s^{m}}{(q ; q)_{m}} \\
& =E(y / x, x ; \theta)\left\{\sum_{n=0}^{\infty} \sum_{m=0}^{\infty}(-1)^{m} q^{\left(\begin{array}{c}
m \\
2
\end{array}\right)} \frac{a^{n} t^{n}}{(q ; q)_{n}} \frac{a^{m} s^{m}}{(q ; q)_{m}}\right\} \\
& =E(y / x, x ; \theta)\left\{\sum_{n=0}^{\infty} \frac{a^{n} t^{n}}{(q ; q)_{n}} \sum_{m=0}^{\infty}(-1)^{m} q^{\left(\begin{array}{c}
m \\
2
\end{array}\right)} \frac{(a s)^{m}}{(q ; q)_{m}}\right\} \quad(|a t|<1) \\
& =E(y / x, x ; \theta)\left\{\frac{(a s ; q)_{\infty}}{(a t ; q)_{\infty}}\right\} .
\end{aligned}
$$

Using (4.4), we complete the proof.

Applying the operator $E(a, b ; \theta)$ one more time, we obtain following triple sum identity.

Theorem 4.4. We have

$$
\begin{aligned}
& \sum_{n=0}^{\infty} \sum_{m=0}^{\infty} \sum_{k=0}^{\infty}(-1)^{k} q^{-\left(\begin{array}{c}
k \\
2
\end{array}\right)-(m+n) k-m n} U_{n+m+k}(x, y, a ; q) \frac{t^{n}}{(q ; q)_{n}} \frac{s^{m}}{(q ; q)_{m}} \frac{v^{k}}{(q ; q)_{k}} \\
& \quad=\frac{(y q / a, a s, a t ; q)_{\infty}}{(x q / a, a v ; q)_{\infty}}{ }_{3} \phi_{2}\left(\begin{array}{c}
y / x, q / a s, q / a t \\
y q / a, q / a v
\end{array} ; q, \frac{x s t}{v}\right)
\end{aligned}
$$

provided that $\max \{|a v|,|x q / a|,|x s t / v|\}<1$. 
Proof. By the operator identity (4.6), the left hand side of (4.8) equals

$$
\begin{aligned}
& E(y / x, x ; \theta)\left\{\sum_{n=0}^{\infty} \sum_{m=0}^{\infty} \sum_{k=0}^{\infty}(-1)^{m+n} q^{\left(\begin{array}{c}
n \\
2
\end{array}\right)+\left(\begin{array}{c}
m \\
2
\end{array}\right)} \frac{(a t)^{n}}{(q ; q)_{n}} \frac{(a s)^{m}}{(q ; q)_{m}} \frac{(a v)^{k}}{(q ; q)_{k}}\right\} \quad(|a v|<1) \\
& =E(y / x, x ; \theta)\left\{\frac{(a s, a t ; q)_{\infty}}{(a v ; q)_{\infty}}\right\} .
\end{aligned}
$$

Applying the operator identity (4.5), we complete the proof.

Setting $s \rightarrow 0$ and $t \rightarrow s, v \rightarrow t$ and applying Jackson's transformation formula [15, III.4], the triple sum (4.8) reduces to the Rogers-type formula (4.7). The Cauchy companion operator also applies to other Rogers-type formulas for the Al-Salam-Carlitz polynomials, including the one given in the previous section. Moreover, we can also derive the following Rogers-type formula

$$
\begin{aligned}
\sum_{n=0}^{\infty} \sum_{m=0}^{\infty} q^{-m n} U_{n+m}(x, y, a ; q) & \frac{t^{n}}{(q ; q)_{n}} \frac{s^{m}}{(q ; q)_{m}} \\
= & \frac{(y q / a, a s, a t ; q)_{\infty}}{(x q / a ; q)_{\infty}}{ }_{3} \phi_{1}\left(\begin{array}{c}
y / x, q / a s, q / a t \\
y q / a
\end{array} ; q, \frac{a x s t}{q}\right)
\end{aligned}
$$

where $\max \{|x q / a|,|a x s t / q|\}<1$. It should be noticed that the above formula is not a consequence of the Rogers formula for the bivariate Rogers-Szegö polynomials $h_{n}(x, y \mid q)[13$, Theorem 3.1] by replacing $q$ with $q^{-1}$.

We now present Mehler's formula without the terminating condition.

Theorem 4.5. We have

$$
\begin{aligned}
& \sum_{n=0}^{\infty}(-1)^{n} q^{-\left(\begin{array}{c}
n \\
2
\end{array}\right)} U_{n}(x, y, a ; q) U_{n}(u, v, b ; q) \frac{t^{n}}{(q ; q)_{n}} \\
& \quad=\frac{(y q / a, a b t, a v t ; q)_{\infty}}{(x q / a, a u t ; q)_{\infty}}{ }_{3} \phi_{2}\left(\begin{array}{c}
y / x, q / a b t, q / a v t \\
y q / a, q / a u t
\end{array} ; q, \frac{x b v t}{u}\right)
\end{aligned}
$$

provided that $\max \{\mid$ aut $|| x, q / a|| x b v t / u \mid\}<$,1 .

Proof. Using (4.6) and (1.3), we find

$$
\begin{aligned}
& \sum_{n=0}^{\infty}(-1)^{n} q^{-\left(\begin{array}{c}
n \\
2
\end{array}\right)} U_{n}(x, y, a ; q) U_{n}(u, v, b ; q) \frac{t^{n}}{(q ; q)_{n}} \\
& \quad=E(y / x, x ; \theta)\left\{\sum_{n=0}^{\infty} U_{n}(u, v, b ; q) \frac{(a t)^{n}}{(q ; q)_{n}}\right\} \quad(|a u t|<1) \\
& \quad=E(y / x, x ; \theta)\left\{\frac{(a v t ; a b t ; q)_{\infty}}{(a u t ; q)_{\infty}}\right\} .
\end{aligned}
$$

So the proof is completed by using the operator identity (4.5).

Comparing the above Mehler's formula with the terminating form (3.10), it leads to the following transformation formula for ${ }_{3} \phi_{2}$ series. 
Corollary 4.5.2. We have

$$
{ }_{3} \phi_{2}\left(\begin{array}{c}
y / x, q / a b t, q / a v t \\
y q / a, q / a u t
\end{array} ; q, \frac{x b v t}{u}\right)=\frac{(y b t, x q / a ; q)_{\infty}}{(x b t, y q / a ; q)_{\infty}} \phi_{2}\left(\begin{array}{c}
y / x, v / u, q / a b t \\
q / x b t, q / a u t
\end{array} ; q, q\right),
$$

provided that $v / u=q^{-r}$ for a nonnegative integer $r$.

\section{Generating Functions for Products of $U_{n}(x, y, a ; q)$}

The objective of this section is to give several generating function formulas for products of the Al-Salam-Carlitz polynomials by using the Cauchy companion operator. Keep in mind that the Al-Salam-Carlitz polynomials are extensions of the Rogers-Szegö polynomials defined by

$$
g_{n}(a \mid q)=\sum_{k=0}^{n}\left[\begin{array}{l}
n \\
k
\end{array}\right] q^{k(k-n)} a^{k} .
$$

It is easily seen that

$$
U_{n}(0,1, a ; q)=(-1)^{n} q^{\left(\begin{array}{c}
n \\
2
\end{array}\right)} g_{n}(a \mid q)
$$

Theorem 5.1. We have

$$
\begin{gathered}
\sum_{n=0}^{\infty} \sum_{m=0}^{\infty}(-1)^{n+m} q^{-\left(\begin{array}{c}
n+m \\
2
\end{array}\right)} U_{n+m}(x, y, a ; q) U_{n}(u, v, b ; q) U_{m}(z, w, c ; q) \frac{t^{n}}{(q ; q)_{n}} \frac{s^{m}}{(q ; q)_{m}} \\
\quad=\frac{(y q / a, a b t, a v t, a c s, a w s ; q)_{\infty}}{(x q / a, a u t, a z s ; q)_{\infty}} 5 \phi_{3}\left(\begin{array}{c}
y / x, q / a b t, q / a v t, q / a c s, q / a w s \\
y q / a, q / a u t, q / a z s
\end{array} ; q, \frac{x a b c v w t s}{u z q}\right),
\end{gathered}
$$

provided that $\max \{|x q / a|, \mid$ aut $|$,$| azs \mid\}<1$.

Proof. By the operator identity (4.6) acting on the parameter $a$, we obtain

$$
\begin{aligned}
& \sum_{n=0}^{\infty} \sum_{m=0}^{\infty}(-1)^{n+m} q^{-\left(\begin{array}{c}
n+m \\
2
\end{array}\right)} U_{n+m}(x, y, a ; q) U_{n}(u, v, b ; q) U_{m}(z, w, c ; q) \frac{t^{n}}{(q ; q)_{n}} \frac{s^{m}}{(q ; q)_{m}} \\
& \quad=E(y / x, x ; \theta)\left\{\sum_{n=0}^{\infty} \sum_{m=0}^{\infty} U_{n}(u, v, b ; q) U_{m}(z, w, c ; q) \frac{(a t)^{n}}{(q ; q)_{n}} \frac{(a s)^{m}}{(q ; q)_{m}}\right\} .
\end{aligned}
$$

Employing the generating function (1.3) with $\max \{|a u t|,|a z s|\}<1$ and the operator identity (4.2) with $|x q / a|<1$, we see that (5.1) equals

$$
\begin{aligned}
& E(y / x, x ; \theta)\left\{\frac{(a b t, a v t ; q)_{\infty}(a c s, a w s ; q)_{\infty}}{(a u t ; q)_{\infty}(a z s ; q)_{\infty}}\right\} \\
& \quad=\frac{(y q / a ; q)_{\infty}}{(x q / a ; q)_{\infty}} \sum_{k=0}^{\infty} \frac{(y / x ; q)_{k} q^{\left(\begin{array}{c}
k \\
2
\end{array}\right)}}{(q, y q / a ; q)_{k}}\left(-\frac{x q}{a}\right)^{k} \frac{\left(a b t q^{-k}, a v t q^{-k}, a c s q^{-k}, a w s q^{-k} ; q\right)_{\infty}}{\left(a u t q^{-k}, a z s q^{-k} ; q\right)_{\infty}}
\end{aligned}
$$

as desired. This completes the proof.

Setting $x, u, z \rightarrow 0$ and $y, v, w \rightarrow 1$, the above theorem reduces to the following generating function formula for the Rogers-Szegö polynomials $g_{n}(x \mid q)$. 
Theorem 5.2. We have

$$
\begin{aligned}
& \sum_{n=0}^{\infty} \sum_{m=0}^{\infty}(-1)^{n+m} q^{\left(\begin{array}{c}
n \\
2
\end{array}\right)+\left(\begin{array}{c}
m \\
2
\end{array}\right)} g_{n+m}(a \mid q) g_{n}(b \mid q) g_{m}(c \mid q) \frac{t^{n}}{(q ; q)_{n}} \frac{s^{m}}{(q ; q)_{m}} \\
& =(q / a, a t, a b t, a s, a c s ; q)_{\infty}{ }_{4} \phi_{1}\left(\begin{array}{c}
q / a t, q / a b t, q / a s, q / a c s \\
q / a
\end{array} ; q, \frac{a^{3} b c t^{2} s^{2}}{q^{3}}\right) .
\end{aligned}
$$

It should be noted that Cao has considered the same generating function and obtained a double summation formula, see [7, Theorem 4.4]. Using similar arguments, we can derive several other generating function formulas for products of $U_{n}(x, y, a ; q)$. The detailed proofs are omitted.

Theorem 5.3. Assume $\max \{|x q / a|, \mid$ aut $\mid\}<1$. We have

$$
\begin{aligned}
& \sum_{n=0}^{\infty}(-1)^{n+m} q^{-\left(\begin{array}{c}
n+m \\
2
\end{array}\right)} U_{n+m}(x, y, a ; q) U_{n}(u, v, b ; q) \frac{t^{n}}{(q ; q)_{n}} \\
& \quad=\frac{(y q / a, a b t, a v t ; q)_{\infty}}{(x q / a, a u t ; q)_{\infty}} a^{m}{ }_{3} \phi_{2}\left(\begin{array}{c}
y / x, q / a b t, q / a v t \\
y q / a, q / a u t
\end{array} ; q, \frac{x b v t}{u q^{m}}\right) .
\end{aligned}
$$

Letting $x, u \rightarrow 0, y, v \rightarrow 1$ and applying the transformation formula for ${ }_{2} \phi_{1}$ series [15, Appendix III.2], we are led to the following formula due to Cao [7, Theorem 4.1],

$$
\begin{aligned}
& \sum_{n=0}^{\infty}(-1)^{n} q^{\left(\begin{array}{c}
n \\
2
\end{array}\right)} g_{n+m}(a \mid q) g_{n}(b \mid q) \frac{t^{n}}{(q ; q)_{n}} \\
& \quad=\frac{(a b t, a t, b t, t ; q)_{\infty}}{\left(a b t^{2} / q ; q\right)_{\infty}} \frac{(q / t ; q)_{m}}{(b t / q)^{m}\left(q^{2} / a b t^{2} ; q\right)_{m}}{ }_{2} \phi_{1}\left(\begin{array}{c}
q^{-m}, q / a b t \\
t q^{-m}
\end{array} ; q, b t\right) .
\end{aligned}
$$

Theorem 5.4. Assume $\max \{|x q / a|,|u q / b|\}<1$. We have

$$
\begin{gathered}
\sum_{n=0}^{\infty} \sum_{m=0}^{\infty} \sum_{k=0}^{\infty}(-1)^{k} q^{-\left(\begin{array}{c}
k \\
2
\end{array}\right)-(n+m) k} U_{n+k}(x, y, a ; q) U_{m+k}(u, v, b ; q) \frac{t^{n}}{(q ; q)_{n}} \frac{s^{m}}{(q ; q)_{m}} \frac{z^{k}}{(q ; q)_{k}} \\
=\frac{(y q / a, v q / b, b s, a t, a b z ; q)_{\infty}}{(x q / a, u q / b ; q)_{\infty}} \sum_{k=0}^{\infty} \frac{(-1)^{k} q^{-\left(\begin{array}{c}
k \\
2
\end{array}\right)(y / x, q / a t, q / a b z ; q)_{k}}\left(\frac{x a b t z}{q}\right)^{k}}{(q, y q / a ; q)_{k}} \\
\times{ }_{3} \phi_{1}\left(\begin{array}{c}
v / u, q / b s, q^{k+1} / a b z \\
v q / b
\end{array} ;, \frac{a b u s z}{q^{k+1}}\right) .
\end{gathered}
$$

Setting $x, u \rightarrow 0, y, v \rightarrow 1$, we obtain

$$
\begin{aligned}
& \sum_{n=0}^{\infty} \sum_{m=0}^{\infty} \sum_{k=0}^{\infty}(-1)^{n+m+k} q^{\left(\begin{array}{c}
n \\
2
\end{array}\right)+\left(\begin{array}{c}
m \\
2
\end{array}\right)+\left(\begin{array}{c}
k \\
2
\end{array}\right)} g_{n+k}(a \mid q) g_{m+k}(b \mid q) \frac{t^{n}}{(q ; q)_{n}} \frac{s^{m}}{(q ; q)_{m}} \frac{z^{k}}{(q ; q)_{k}} \\
& \quad=(q / a, q / b, b s, a t, a b z ; q)_{\infty} \sum_{k=0}^{\infty} \frac{(q / a t, q / a b z ; q)_{k}}{(q, q / a ; q)_{k}}\left(\frac{a b t z}{q}\right)^{k}{ }_{2} \phi_{1}\left(\begin{array}{c}
q / b s, q^{k+1} / a b z \\
q / b
\end{array} ; q, \frac{a b s z}{q^{k+1}}\right) .
\end{aligned}
$$


Because of the convergence requirement, we should assume that $q / a t=q^{-r}$ and $\left|a b s z / q^{r+1}\right|<$ 1. Under this condition, the ${ }_{2} \phi_{1}$ series in the above expression can be summed by the $q$-Gauss formula [15, Appendix (II.8)]. It follows that

$$
\begin{gathered}
\sum_{n=0}^{\infty} \sum_{m=0}^{\infty} \sum_{k=0}^{\infty}(-1)^{n+m+k} q^{\left(\begin{array}{c}
n \\
2
\end{array}\right)+\left(\begin{array}{c}
m \\
2
\end{array}\right)+\left(\begin{array}{c}
k \\
2
\end{array}\right)} g_{n+k}(a \mid q) g_{m+k}(b \mid q) \frac{t^{n}}{(q ; q)_{n}} \frac{s^{m}}{(q ; q)_{m}} \frac{z^{k}}{(q ; q)_{k}} \\
=\frac{(q / a, s, a t, a z, b s, a b z ; q)_{\infty}}{(a b s z / q ; q)_{\infty}} \phi_{2}\left(\begin{array}{c}
q^{-n}, q / a z, q / a b z \\
q / a, q^{2} / a b s z
\end{array} ; q, \frac{a t z}{s}\right) .
\end{gathered}
$$

It should mentioned that the terminating condition $t / v=q^{-r}$ is overlooked in the operator identity of Zhang and Wang [19, Theorem 2.5], and the same condition is required concerning the identity of Cao [7, Eq. (2.9)].

Theorem 5.5. Assume $\max \{|x q / a|,|v q / b|\}<1$. We have

$$
\begin{aligned}
& \sum_{k=0}^{\infty}(-1)^{m+n+k} q^{-\left(\begin{array}{c}
n+k \\
2
\end{array}\right)-\left(\begin{array}{c}
m \\
2
\end{array}\right)-m k} U_{n+k}(x, y, a ; q) U_{m+k}(u, v, b ; q) \frac{z^{k}}{(q ; q)_{k}} \\
& \quad=\frac{(y q / a, v q / b, a b z ; q)_{\infty}}{(x q / a, u q / b ; q)_{\infty}} a^{n} b^{m} \sum_{k=0}^{\infty} \frac{(y / x, q / a b z ; q)_{k}}{(q, y q / a ; q)_{k}}\left(\frac{x b z}{q^{n}}\right)^{k}{ }_{2} \phi_{1}\left(\begin{array}{c}
v / u, q^{k+1} / a b z \\
v q / b
\end{array} ; q, \frac{a u z}{q^{m+k}}\right) .
\end{aligned}
$$

Setting $x, u \rightarrow 0, y, v \rightarrow 1$, we deduce that

$$
\begin{aligned}
& \sum_{k=0}^{\infty}(-1)^{k} q^{\left(\begin{array}{c}
k \\
2
\end{array}\right)} g_{n+k}(a \mid q) g_{m+k}(b \mid q) \frac{z^{k}}{(q ; q)_{k}} \\
& \quad=(q / a, q / b, a b z ; q)_{\infty} a^{n} b^{m} \sum_{k=0}^{\infty} \frac{(-1)^{k} q^{\left(\begin{array}{c}
k \\
2
\end{array}\right)(q / a b z ; q)_{k}}}{(q, q / a ; q)_{k}}\left(\frac{b z}{q^{n}}\right)^{k} \phi_{1}\left(\begin{array}{c}
q^{k+1} / a b z \\
q / b
\end{array} ; q, \frac{a z}{q^{m+k}}\right),
\end{aligned}
$$

which can be deduced from the formula of Cao [7, Theorem 4.3] by three transformations, namely, the the limiting case of [15, Appendix (III.2)] when $c \rightarrow 0$, the two transformations [15, Appendix (III.2)] and [15, Appendix(III.7)].

Acknowledgments. This work was supported by the 973 Project, the PCSIRT Project of the Ministry of Education, and the National Science Foundation of China.

\section{References}

[1] W.H. Abdi, A basic analogue of the Bessel polynomials, Math. Nachr. 30 (1965) 209-219.

[2] W. Al-Salam and L. Carlitz, Some orthogonal q-polynomials, Math. Nachr. 30 (1965) $47-61$.

[3] G.E. Andrews, On the foundations of combinatorial theory, V: Eulerian differential operators, Stud. Appl. Math. 50 (1971) 345-375.

[4] R. Askey and S.K. Suslov, The $q$-harmonic oscillator and the Al-Salam and Carlitz polynomials, Lett. Math. Phys. 29 (1993) 123-132. 
[5] R. Askey and J. Wilson, Some Basic Hypergeometric Orthogonal Polynomials that Generalize Jacobi Polynomials, Mem. Amer. Math. Soc. 54 (1985) no. 319.

[6] N.M. Atkishiyev and S.M. Nagiyev, On Rogers-Szegö polynomials, J. Phys. A: Math. Gen. 27 (1994) L611-L615.

[7] J. Cao, New proofs of generating functions for Rogers-Szegö polynomials, Appl. Math. Comput. 207 (2009) 486-492.

[8] L. Carlitz, Generating functions for certain q-orthogonal polynomials, Collectanea Math. 23 (1972) 91-104.

[9] V.Y.B. Chen, q-Difference Operator and Basic Hypergeometric Series, Ph.D. Thesis, Nankai University, Tianjin, China, 2009.

[10] V.Y.B. Chen and N.S.S. Gu, The Cauchy operator for basic hypergeometric series, Adv. Appl. Math. 41 (2008) 177-196.

[11] W.Y.C. Chen, A.M. Fu and B.Y. Zhang, The homogeneous $q$-difference operator, Adv. Appl. Math. 31 (2003) 659-668.

[12] W.Y.C. Chen and Z.G. Liu, Parameter augementing for basic hypergeometric series, II, J. Combin. Theory, Ser. A 80 (1997) 175-195.

[13] W.Y.C. Chen, H.L. Saad and L.H. Sun, The bivariate Rogers-Szegö polynomials, J. Phys. A: Math. Theor. 40 (2007) 6071-6084.

[14] J.P Fang, q-Difference operator identities and applications, J. Math. Anal. Appl. 332 (2007) 1393-1407.

[15] G. Gasper and M. Rahman, Basic Hypergeometric Series, 2nd Ed., Cambridge University Press, Cambridge, MA, 2004.

[16] D. Kim, On Combinatorics of Al-Salam Carlitz polynomials, European J. Combin. 18 (1997) 295-302.

[17] R. Koekoek and R.F. Swarttouw, The Askey-Scheme of Hypergeometric Orthogonal Polynomials and its $q$-Analogue, Report no. 98-17, Delft University of Technology, 1998, http://aw.twi.tudelft.nl/ koekoek/askey.html.

[18] S. Roman, More on the umbral calculus, with emphasis on the $q$-umbral calculus, $J$. Math. Anal. Appl. 107 (1985) 222-254.

[19] Z.Z. Zhang and J. Wang, Two operator identities and their applications to terminating basic hypergeometric series and q-integrals, J. Math. Anal. Appl. 312 (2005) 653-665. 